\title{
Felodipine-associated gingival overgrowth in a type 2 diabetic patient: A case report and literature review
}

\author{
LIN SUN $^{1}$, CHENGXIN WANG $^{2}$, SHUGANG XI $^{1}$, TONG ZHOU ${ }^{1}$, GUIXIA WANG ${ }^{1}$ and XIAOKUN GANG ${ }^{1}$ \\ Departments of ${ }^{1}$ Endocrinology and Metabolism, and ${ }^{2}$ Developmental and Behavior Pediatrics, \\ The First Hospital of Jilin University, Changchun, Jilin 130021, P.R. China
}

Received August 11, 2018; Accepted February 4, 2019

DOI: $10.3892 /$ etm.2019.7376

\begin{abstract}
Gingival overgrowth (GO) includes gingival enlargement and hyperplasia and may be induced by certain drugs, including calcium channel blockers (CCBs), particularly first-generation CCBs. However, to date, only few cases of GO induced by second- or third-generation CCBs have been reported. The present study reports on a case of a 48 -year-old diabetic male who was admitted to the First Hospital of Jilin University (Changchun, China) due to poor blood glucose control. This patient was diagnosed with GO. Review of the patient's medical history revealed diagnoses of type 2 diabetes and hypertension, as well as the use of felodipine, a second-generation CCB, to control hypertension. The hypertensive drugs were replaced and the new drugs helped the patient control his blood glucose levels. Additionally, the patient was instructed on methods he could use to improve his oral hygiene, including rinsing of the teeth following each meal and increasing the frequency of tooth brushing per day. After 3 months, the clinical symptoms of GO were relieved. The relevant literature was also reviewed to gain an improved understanding of the correlation between $\mathrm{GO}$ and $\mathrm{CCBs}$, as well as diabetes and poor oral hygiene.
\end{abstract}

\section{Introduction}

Gingival overgrowth (GO), which usually occurs at the upper and lower anterior teeth, includes gingival enlargement and hyperplasia. GO is characterized by a change in the color of the gingiva, bleeding upon probing, compromised appearance of the teeth, and difficulties regarding mastication, deglutition and speech (1-5). The pathogenesis of GO is thought to be

Correspondence to: Dr Guixia Wang or Dr Xiaokun Gang, Department of Endocrinology and Metabolism, The First Hospital of Jilin University, 71 Xinmin Road, Changchun, Jilin 130021, P.R. China

E-mail: gwang168_1@163.com

E-mail: insightful@126.com

Key words: calcium channel blocker, felodipine, drug-induced gingival overgrowth multifactorial, involving inflammation, malignancy (e.g., oral cavity tumor or other solid tumor type), systemic diseases and undesired effects associated with systemic administration of certain drugs $(6,7)$. GO that occurs via the latter mechanism is referred to as drug-induced gingival overgrowth (DIGO) and has a prevalence of $3-20 \%$ (8). To date, $>20$ drugs have been reported to be associated with DIGO, including anti-convulsants (e.g., phenytoin), immunosuppressants (e.g., cyclosporine A) and calcium channel blockers (CCBs, e.g., nifedipine and verapamil) (9-22). CCBs are administered to treat various cardiac diseases, including hypertension, angina pectoris and certain arrhythmias (e.g., supraventricular tachycardia) and exert their effect on voltage-dependent $\mathrm{Ca}^{2+}$ channels in smooth muscles (23), subsequently promoting a higher concentration of extracellular calcium and decreasing the level of intracellular calcium. The changes in calcium concentrations inside and outside of cells affect the activation of collagenase and processes involved in apoptosis (24-26). Therefore, CCBs have an important role in the initiation and progression of tissue overgrowth.

CCBs are primarily divided into three categories: Phenylalkylamine derivatives (e.g., verapamil), benzothiazepine derivatives (e.g., diltiazem) and substitute dihydropyridines (e.g., nifedipine, amlodipine, felodipine, isradipine, nicardipine, nimodipine, oxodipine, nisodipine and nitrendipine) (23). Nifedipine, as a first-generation dihydropyridine, has been widely used in the management of cardiac diseases, including hypertension. In the mid-1980s, Lombardi et al (27) first reported on a case of GO caused by nifedipine. Since then, further cases of GO linked to the use of nifedipine have been reported. The incidence of nifedipine-induced $\mathrm{GO}$ varies among different studies, ranging from $14-83 \%$ (28). Compared to the first-generation CCBs, the involvement of second- and third-generation dihydropyridines, including felodipine and amlodipine, in the pathogenesis of DIGO has been less frequently reported. One study reported on incidence of amlodipine-induced GO of 1.4-3.3\% (8). Felodipine was first reported to cause DIGO in 1991 (27), and Fay et al (4) reported on one case of felodipine-associated GO in a patient with type 2 diabetes, whose histological characteristics of GO were similar to those in DIGO and withdrawal of felodipine almost fully resolved the GO. The present study reports on a case of GO that was attributed to felodipine treatment. To the best of our knowledge, the present study is the first to report on felodipine-induced GO in a Chinese patient with type 2 diabetes. 


\section{Case report}

A 48-year-old man from Jilin Province in the northeast region of China presented at the Endocrinology Department of the First Clinical Hospital of Jilin University (Changchun, China) with the chief complaint of poor blood glucose control lasting for nearly 3 days. The patient denied a family history of hypertension and diabetes mellitus but admitted that his blood pressure had been high for 4 years, with a peak blood pressure of 180/100 $\mathrm{mmHg}$. Various anti-hypertensive drugs had been used to control his blood pressure but had a poor efficiency. The patient had a history of diabetes of $\sim 1$ year. Aside from diet control, he did not take any medications or insulin to control his blood glucose levels and monitored his fingertip blood glucose regularly. At 3 days prior to presentation, his fingertip blood glucose were $14 \mathrm{mmol} / \mathrm{l}$ and glycated hemoglobin (HbAlc) levels were $8.4 \%$, which prompted him to visit our department. On physical examination, the patient had a body temperature of $36.5^{\circ} \mathrm{C}$, heart rate of 88 beats $/ \mathrm{min}$, respiratory rate of 20 breaths $/ \mathrm{min}$ and blood pressure of $160 / 100 \mathrm{mmHg}$. He was well developed and moderately nourished with a body mass index of $26.2 \mathrm{~kg} / \mathrm{m}^{2}$. His skin and sclera had no yellow staining. The trachea was in the midline and the thyroid was not enlarged. No abnormal breath or heart sound was noted on auscultation. No abdominal positive signs were identified. Routine examination indicated the following: Normal liver and kidney function, fasting blood glucose of $7.9 \mathrm{mmol} / 1$, blood potassium of $3.1 \mathrm{mmol} / 1$ (normal range, 3.5-5.3 mmol/l), carbon dioxide binding capacity of $35.6 \mathrm{mmol} / \mathrm{l}$ (normal range, 22-30 mmol/l) and no abnormalities in electrocardiogram and chest X-ray. Abdominal computed tomography indicated slight hyperplasia of the bilateral adrenal grands and thick insides and thin outsides of limbs. No retinopathy or diabetic peripheral neuropathy was observed.

On physical examination, GO was identified. The patient had mild gingival soreness when chewing hard foods, but did not have any difficulties with mastication. Review of the patient's medical history revealed the use of oral medicines to control hypertension. He had started taking a combination of irbesartan, an angiotensin II receptor antagonist, and felodipine $\sim 4$ years previously, but after $\sim 6$ months on this medication, he discontinued irbesartan due to the increasing incidence of hypopiesia. Since then, he had taken only felodipine for $\sim 3.5$ years. He mentioned that GO first occurred 8 months previously while he was using felodipine. Oral examination revealed a mouth opening index of III and GO throughout all quadrants, which was particularly pronounced under the dental papilla (Fig. 1). In addition, the gums bled when probed. All teeth were dark brown due to poor oral hygiene. Based on the patient's medical history and consultation with a dental professional, it is likely that GO in this patient was caused by felodipine. Therefore, felodipine was first replaced with a combination of spironolactone (a diuretic) and terazosin (an $\alpha$-receptor blocker) to control the hypertension. It was then proposed that the patient retains his original dietary habits combined with the use of metformin (a hypoglycemic drug) to reduce blood glucose levels. Finally, the patient was instructed on methods to improve his oral hygiene, including rinsing of the teeth after each meal and increasing the frequency of tooth brushing per day. At the 3-month follow-up, the patient had a blood pressure ranging from $130 / 85$ to $140 / 90 \mathrm{mmHg}$, fasting blood glucose of $7-8 \mathrm{mmol} / 1$ and postprandial blood glucose of $9-10 \mathrm{mmol} / \mathrm{l}$. The level of HbAlc had decreased to $7.5 \%$. Although the GO had not been completely eliminated, the symptoms of tooth soreness disappeared after the triggers of GO were eliminated (Fig. 2).

\section{Discussion}

Felodipine was first reported to cause DIGO in 1991 (27). However to the best of our knowledge, felodipine-induced GO in a Chinese patient with diabetes mellitus has not been previously reported. The present study reports on one case of felodipine-associated $\mathrm{GO}$ in a type 2 diabetic patient.

Although it is well established that CCBs are associated with the development of GO, the exact underlying mechanisms remain to be fully elucidated. Inflammatory as well as non-inflammatory pathways have been indicated to be involved in CCB-induced GO (28). The non-inflammatory pathway includes the upregulation of keratinocyte growth factor, which promotes epithelial cell growth in the oral cavity and matrix synthesis around gingival connected tissues. Such increased matrix synthesis leads to defective collagenase activity attributed to the reduced uptake of folic acid, thereby resulting in the accumulation of connective tissue and development of GO (28).

On the other hand, the inflammatory pathway also has an important role in the interaction between drugs and fibroblasts (28). First, the higher drug concentration in crevicular gingival fluid, which was identified to be up to 292-fold of that detected in serum, may have direct toxic effects. This drug-induced inflammation causes an upregulation of the levels of several cytokine factors, including fibroblast growth factor- 2 , transforming growth factor- $\beta 1$, interleukin-6 (IL-6), IL-1B and platelet derived growth factor- $\beta$ to generate excessive fibroblasts, which contributes to the development of fibrotic gingival hyperplasia $(28,29)$. In addition, the pro-inflammatory cytokines released from inflammatory corpuscles participate in inflammation-associated local cellular events, including mast cell migration, fibroblast proliferation, as well as an increase in extracellular matrix synthesis and decrease in degradation. These processes may create a vicious cycle to promote the pathogenesis of DIGO. Mechanistically, the CCB-induced fluctuation in intra- and extra-cellular calcium levels is mediated via inflammatory signaling (30). It has been reported that a high intracellular free $\mathrm{Ca}^{2+}$ concentration potentiates the activity of growth factors and cell cycle regulators, and thus, promotes cell proliferation and collagen synthesis (30).

Apart from certain drugs, GO has been reported to be associated with other factors, including diabetes. For instance, Van Dis et al (31) reported on four cases of hyperplastic gingival enlargement in diabetes mellitus patients and Fay et al (4) reported one case of felodipine-influenced GO in a patient with uncontrolled type 2 diabetes mellitus. Hence, diabetes mellitus may be regarded as one of the contributing factors to the development of GO, particularly CCB-induced GO. The exact mechanisms by which 


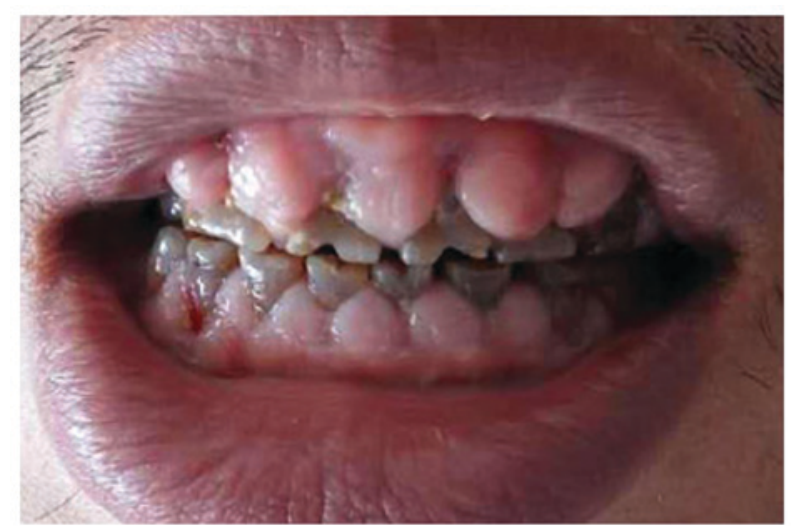

Figure 1. At presentation, all of the patient's teeth were dark brown due to poor oral hygiene. Oral examination revealed a mouth opening index of III and gingival overgrowth throughout all quadrants that was particularly pronounced under the dental papilla.

hyperglycemia contributes to the development of $\mathrm{GO}$ remain elusive. It may be speculated that hyperglycemia is associated with an elevated local inflammatory response, which may have a role in the pathogenesis of GO. Furthermore, the clinical manifestations in previously reported cases of GO included erythematous and edematous hyperplastic gingival tissues, numerous food sediments and severe periodontitis $(32,33)$, all of which were accompanied by poor oral hygiene (34). Therefore, it may be reasoned that the poor gingival hygienic conditions of the present case served as another contributing factor to DIGO (35). However, how these contributing factors act synergistically remains elusive.

The case of the present study had been diagnosed with type 2 diabetes 1 year previously and had experienced poor blood glucose control for 3 days. GO occurred at 8 months prior to the patient's presentation at the department of Endocrinology and Metabolism of the First Hospital of Jilin University, and after the replacement of the angiotensin II receptor antagonist with the CCB felodipine. In addition, his gingival hygienic condition was poor. Of note, the degree of severity of GO, drug dosage and the occurrence time of DIGO were similar to those of a previous case reported by Fay et al (4), except for the controlled diabetes mellitus. Hence, it may be concluded that the gingival hygienic status may be regarded as a major promoting factor for felodipine-induced DIGO. As mentioned above, hyperglycemia is likely to be another contributing factor.

Previously, Fay et al (4) and Khzam et al (35) suggested that surgery and application of antibiotics should be considered as the second choice for treating DIGO. Indeed, upon revision of the medication strategy for the present case, i.e., replacement of the CCB with a diuretic, the symptoms of gingival soreness were relieved and GO had been partially eliminated within 3 months. Therefore, removal of the causative factors, including the improvement of oral hygiene, control of blood glucose levels, and most importantly, replacement of the CCB with a different appropriate anti-hypertensive drug, reduced the clinical symptoms and prevented the recurrence of lesions. Indeed, the clinical significance of the present case is that a simple change of the dihydropyridine drug in combination with improvements in the patient's health care (i.e., controlling

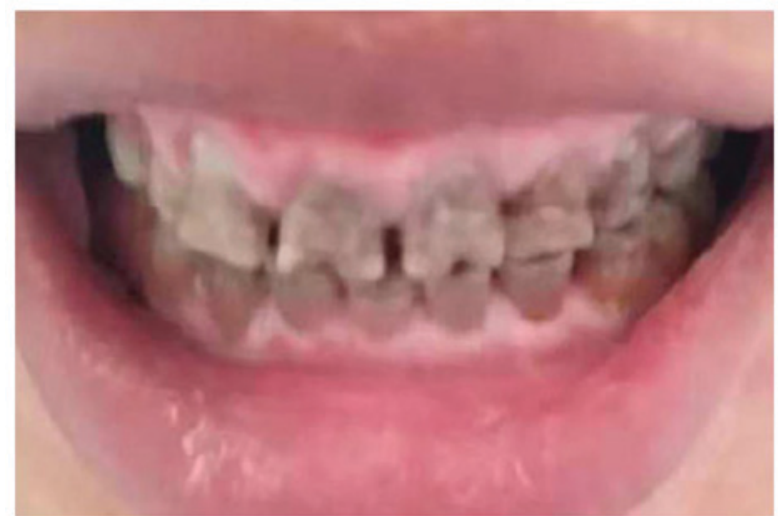

Figure 2. The quality of the patient's teeth was improved after 3 months under the new treatment, although the gingival overgrowth had not been completely eliminated.

diabetes and oral hygiene) permitted the avoidance of surgical intervention.

A literature search did not provide any studies that linked angiotensin II receptor antagonists to the pathogenesis of GO and this possibility was therefore excluded. The present case had recurrent hypokalemia and adrenal hyperplasia. After a series of endocrine system examinations, the patient was determined to have adrenal hyperplasia. In combination with the presence of gingival hyperplasia in the present case, the treatment was switched to aldosterone antagonist spironolactone combined with terazosin anti-hypertensive drug in order to control blood pressure and hypokalemia. From the start of the new treatment, the patient's blood pressure was under control. Trazosin and spironolactone are not known to be associated with drug-induced gingival hyperplasia. The patient was advised to return periodically for follow-up with regard to adrenal hyperplasia and associated endocrine hormone levels.

The limitations of the present case report should be acknowledged. For instance, the local concentration of CCB and glucose around the gingival tissue was not determined and no histological examination was performed. Furthermore, the glucose levels in blood vs. gingival interstitial fluid were not compared, and changes in the gingival tissues by histological examination prior to and following treatment were not determined. These changes may be investigated in further intensive studies.

\section{Acknowledgements}

Not applicable.

\section{Funding}

The present study was sponsored by Jilin Province Science and Technology Development Plan Project (grant nos. 20160623092TC-03 and 20180623083TC-03).

\section{Availability of data and materials}

The datasets used and/or analyzed during the present study are available from the corresponding author on reasonable request. 


\section{Authors' contributions}

GW and XG contributed a lot to the design of the study and revision of the manuscript. LS and $\mathrm{CW}$ wrote the first draft of the manuscript. TZ and SX edited this manuscript and interpreted the data. $\mathrm{LS}, \mathrm{CW}$ and $\mathrm{XG}$ contributed a lot to the acquisition of the data as well as the revision of the final draft. All authors have approved the submitted version and agreed to be accountable for all aspects of the manuscript.

\section{Ethics approval and consent to participate}

The present study was approved by the Ethics Committee of the First Hospital of Jilin University (Changchun, China).

\section{Patient consent for publication}

The patient provided written informed consent for the publication of his data and images in the present study.

\section{Competing interests}

The authors declare that they have no competing interests.

\section{References}

1. Nyska A, Shemesh M, Tal H and Dayan D: Gingival hyperplasia induced by calcium channel blockers: Mode of action. Med Hypotheses 43: 115-118, 1994.

2. Lafzi A, Farahani RM and Shoja MA: Amlodipine-induced gingival hyperplasia. Med Oral Patol Oral Cir Bucal 11: E480-E482, 2006.

3. Joshi S and Bansal S: A rare case report of amlodipine-induced gingival enlargement and review of its pathogenesis. Case Rep Dent 2013: 138248, 2013

4. Fay AA, Satheesh K and Gapski R: Felodipine-influenced gingival enlargement in an uncontrolled type 2 diabetic patient. J Periodontol 76: 1217, 2005.

5. Sucu M, Yuce M and Davutoglu V: Amlodipine-induced massive gingival hypertrophy. Can Fam Physician 57: 436-437, 2011.

6. Hallmon WW and Rossmann JA: The role of drugs in the pathogenesis of gingival overgrowth. A collective review of current concepts. Periodontol 2000 21: 176-196, 1999.

7. Seymour RA, Thomason JM and Ellis JS: The pathogenesis of drug-induced gingival overgrowth. J Clin Periodontol 23 : $165-175,1996$

8. Ono M, Tanaka S, Takeuchi R, Matsumoto H, Okada H, Yamamoto H, Makiyama Y, Hirayama T, Sakamaki T, Fujii A and Akimoto Y: Prevalence of Amlodipine-induced Gingival Overgrowth. Int J Oral-Med Sci 9: 96-100, 2010.

9. Hassell TM and Gilbert GH: Phenytoin sensitivity of fibroblasts as the basis for susceptibility to gingival enlargement. Am J Pathol 112: 218-223, 1983.

10. Casetta I, Granieri E, Desiderá M, Monetti VC, Tola MR, Paolino E, Govoni V and Calura G: Phenytoin-induced gingival overgrowth: A community-based cross-sectional study in Ferrara, Italy. Neuroepidemiology 16: 296-303, 1997.

11. Brunsvold $\mathbf{M}$, Tomasovic J and Ruemping D: The measured effect of phenytoin withdrawal on gingival hyperplasia in children. ASDC J Dent Child 52: 417-421, 1985.

12. Rateitschak-Plüss EM, Hefti A, Lörtscher R and Thiel G: Initial observation that cyclosporin-A induces gingival enlargement in man. J Clin Periodontol 10: 237-246, 1983.

13. Pisanty S, Rahamim E, Ben-Ezra D and Shoshan S: Prolonged systemic administration of cyclosporin A affects gingival epithelium. J Periodontol 61: 138-141, 1990.
14. Daly CG: Resolution of cyclosporin A (CsA)-induced gingival enlargement following reduction in CsA dosage. J Clin Periodontol 19: 143-145, 1992.

15. Barak S, Engelberg IS and Hiss J: Gingival hyperplasia caused by nifedipine. Histopathologic findings. J Periodontol 58: 639-642, 1987.

16. Romanos GE, Schröter-Kermani C, Hinz N, Herrmann D, Strub JR and Bernimoulin JP: Extracellular matrix analysis of nifedipine-induced gingival overgrowth: Immunohistochemical distribution of different collagen types as well as the glycoprotein fibronectin. J Periodontal Res 28: 10-16, 1993.

17. Miranda J, Brunet L, Roset P, Berini L, Farré M and Mendieta C: Prevalence and risk of gingival enlargement in patients treated with nifedipine. J Periodontol 72: 605-611, 2001.

18. Pernu HE, Oikarinen K, Hietanen $\mathrm{J}$ and Knuuttila $\mathrm{M}$ : Verapamil-induced gingival overgrowth: A clinical, histologic, and biochemic approach. J Oral Pathol Med 18: 422-425, 1989.

19. Miller CS and Damm DD: Incidence of verapamil-induced gingival hyperplasia in a dental population. J Periodontol 63: 453-456, 1992.

20. Mehta AV,ChidambaramB and O'Riordan AC: Verapamil-induced gingival hyperplasia in children. Am Heart J 124: 535-536, 1992.

21. Giustiniani S, Robustelli della Cuna F and Marieni $M$ : Hyperplastic gingivitis during diltiazem therapy. Int J Cardiol 15: 247-249, 1987.

22. Fattore L, Stablein M, Bredfeldt G, Semla T, Moran M and Doherty-Greenberg JM: Gingival hyperplasia: A side effect of nifedipine and diltiazem. Spec Care Dentist 11: 107-109, 1991.

23. Godfraind T: Calcium channel blockers in cardiovascular pharmacotherapy. J Cardiovasc Pharmacol Ther 19: 501-515, 2014.

24. Godfraind T: Mechanisms of action of calcium entry blockers. Fed Proc 40: 2866-2871, 1981.

25. Soward AL, Vanhaleweyk GL and Serruys PW: The haemodynamic effects of nifedipine, verapamil and diltiazem in patients with coronary artery disease. A review. Drugs 32: 66-101, 1986.

26. Bose T, Cieślar-Pobuda A and Wiechec E: Role of ion channels in regulating $\mathrm{Ca}^{2+}$ homeostasis during the interplay between immune and cancer cells. Cell Death Dis 6: e1648, 2015.

27. Lombardi T, Fiore-Donno G, Belser U and Di Felice R Felodipine-induced gingival hyperplasia: A clinical and histologic study. J Oral Pathol Med 20: 89-92, 1991.

28. Thomas $\mathrm{P}$, Tolstoy $\mathrm{R}$ and Duraisingh L: Amlodipine induced gingival hyperplasia: A case report. Int J Basic Clin Pharmacol 805-807, 2015 (In Chinese).

29. Gong Y, Lu J, Ding X and Yu Y: Effect of adjunctive roxithromycin therapy on interleukin- $1 \beta$, transforming growth factor- $\beta 1$ and vascular endothelial growth factor in gingival crevicular fluid of cyclosporine A-treated patients with gingival overgrowth. J Periodontal Res 49: 448-457, 2014.

30. Matsumoto H, Takeuchi R, Ono M, Akimoto Y, Kobayashi N and Fujii A: Drug-induced gingival overgrowth and its tentative pharmacotherapy. Jpn Dent Sci Rev 46: 11-16, 2010.

31. Van Dis ML, Allen CM and Neville BW: Erythematous gingival enlargement in diabetic patients: A report of four cases. J Oral Maxillofac Surg 46: 794-798, 1988.

32. Padmanabhan S and Dwarakanath CD: Severe gingival enlargement associated with aggressive periodontitis. J Indian Soc Periodontol 17: 115-119, 2013.

33. Agrawal AA: Gingival enlargements: Differential diagnosis and review of literature. World J Clin Cases 3: 779-788, 2015

34. Reali L, Zuliani E, Gabutti L, Schönholzer C and Marone C: Poor oral hygiene enhances gingival overgrowth caused by calcineurin inhibitors. J Clin Pharm Ther 34: 255-260, 2009.

35. Khzam N, Bailey D, Yie HS and Bakr MM: Gingival enlargement induced by felodipine resolves with a conventional periodontal treatment and drug modification. Case Rep Dent 2016: 1095927, 2016.

cc) (i) $\ominus$ This work is licensed under a Creative Commons Attribution-NonCommercial-NoDerivatives 4.0 International (CC BY-NC-ND 4.0) License. 